ceuticals, Inc.), in this way the structurally driven optimization of inhibitors can incorporate relevant aspects of drug design.

\section{Clinical trials}

There was very little discussion of clinical trials for structure based drugs, and it appears that at this time none have actually been marketed. However, informal conversations indicated that several drugs which can be legitimately said to have profited from structure-based design, are well into clinical testing. One example is the development of PNP inhibitors designed by Dr Bugg and his collaborators at BioCryst. It was initially hoped that inhibition of this enzyme might allow a selective suppression of T cells, leaving B cells unimpaired. One inhibitor, called BCX-34, is being tested against several targets. Phase I/II clinical trials have been completed with eight patients suffering from cutaneous $\mathrm{T}$-cell lymphoma. Also, a topical formulation of the drug is being tested in Phase I/II trials against allergic contact dermatitis.

Structure-based drug design looks very promising on paper and will hopefully make as big an impact on the practical world of pharmaceuticals as protein crystallography has made on our understanding of biological systems. The next meeting on structure-based drug design may well present clinical results showing the field is living up to, if not beyond, its great promise.

* Symposium on structure-based drug design, 22-25 April 1994, Panama City Beach, Florida.

1. Appelt K. et al. J.med.Chem. 34, 1925 1934 (1991).
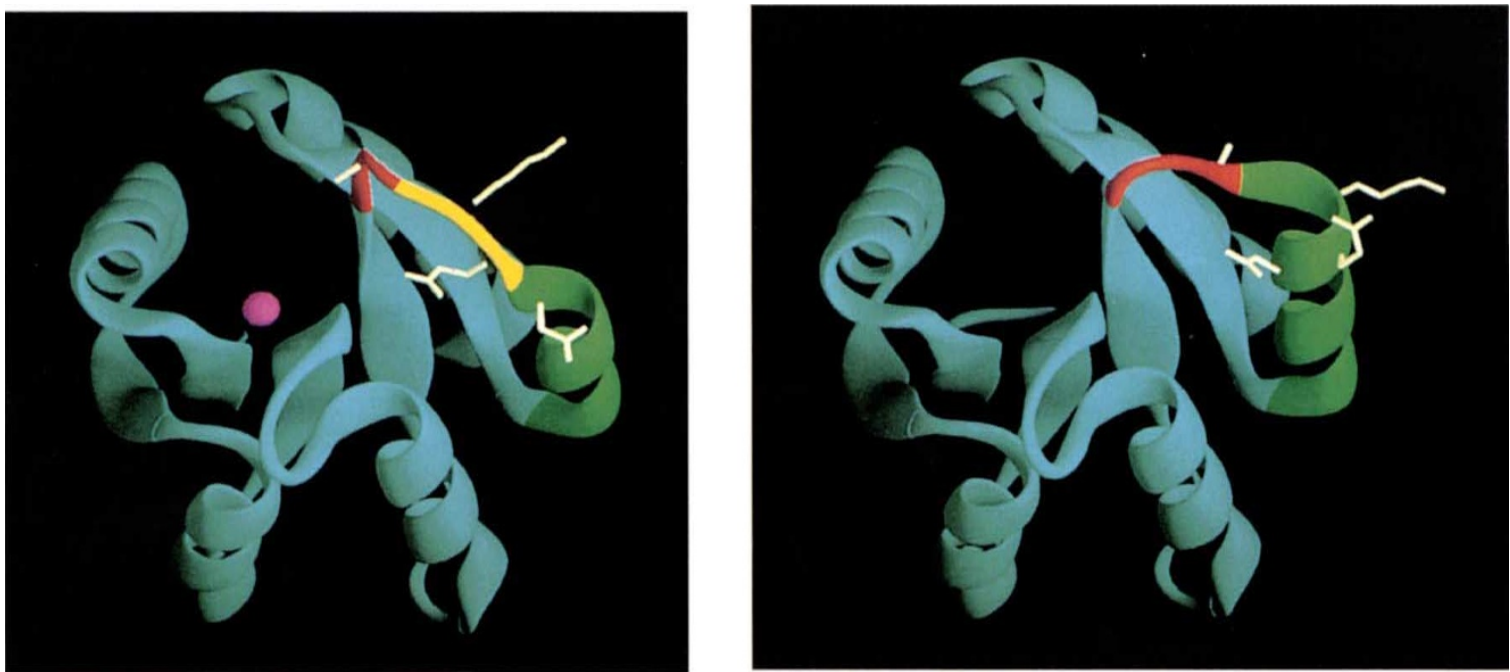

\title{
Turning-on tumbling
}

Regulation of phosphorylation state is a frequent mechanism for control of a protein's activity. It is not enough however simply to state that a protein is active when phosphorylated and inactive when dephosphorylated and consider the process elucidated. At the very least it is necessary for the chemical/ structural changes that are brought about by this phosphorylation to be determined and for their detailed functional effects discovered. With the structure of $C$ heY, a small a/ $B$ protein involved in the chemotactic response of many bacteria, complexed with magnesium (Bellsolell L., Priesto J., Serrano L. \& Coll M. J.molec.Biol. in the press), the first half of this process has been achieved.

In response to adverse environmental conditions CheY becomes phosphorylated by a histidine kinase CheA. Phosphorylated CheY interacts with the flagellar motor altering its direction of rotation and causing the bacterium to change from straight line swimming to a tumbling motion. The presence of magnesium is required for this activity thus comparison of apo- (left figure) and Mg2+-CheY (right figure; $\mathrm{Mg} 2+$ in purple) highlights the changes induced by phosphorylation. There is clearly a dramatic reorganization of part of the molecule: one turn of a three turn a-helix (green) becomes unwound (yellow) producing movements of up to $10 \AA$ in certain residues, extension of a $\beta$-sheet and production of a new B-turn (red). This all serves to modify significantly the proteins surface in a region which has already been shown, by mutational analysis, to be important for CheY activity.

The structural changes observed in CheY may represent a common theme among bacterial response regulators. A number of other systems in bacteria, including those controlling sporulation, nitrogen fixation, virulence and osmoregulation, contain regulators homologous to CheY. In these systems the regulator's target may be different but it is clear that the "on/off switch" is structural rather than chemical. 